\title{
Correlación entre potencia y resistencia en jugadores de fútbol sala de Boyacá
}

\section{Relation between power and resistance in professional futsal players}

\author{
Nelson Enrique Perilla-Sáenz¹ (iD); Milton Javier Pirazán-Rodríguez¹ (iD
}

'Universidad Santo Tomás, Tunja, Boyacá, Colombia.mg12ps@gmail.com; miljapiro1977@hotmail.com.

Cómo citar: Perilla-Sáenz, N.E.; Pirazán-Rodríguez, M.J. 2022. Correlación entre potencia y resistencia en jugadores de fútbol sala de Boyacá. Rev. Digit. Act. Fis. Deport. 8(1):e2155. http://doi.org/10.31910/rdafd.v8.n1.2022.2155

Artículo de acceso abierto publicado por Revista Digital: Actividad Física y Deporte, bajo una licencia Creative Commons CC BY-NC 4.0 Publicación oficial de la Universidad de Ciencias Aplicadas y Ambientales U.D.C.A, Institución de Educación Superior Acreditada de Alta Calidad por el Ministerio de Educación Nacional.

Recibido: agosto 30 de 2021 Aceptado: septiembre 28 de 2021 Editado por: Álvaro José Gracia Díaz

\section{RESUMEN}

Introducción: El futbol sala es un deporte de colaboración - oposición, donde los miembros de un mismo equipo trabajan para conseguir un objetivo $y$, al mismo tiempo, se relacionan con los miembros del equipo contrario; sus acciones se desarrollan en un espacio y tiempo veloz, cambiante y dinámico. Objetivo: Investigar la correlación existente entre la potencia y la resistencia a la velocidad en jugadores profesionales de un equipo de Fútbol Sala. Metodología: Se realizó un enfoque empírico analítico y un diseño descriptivo correlacional no experimental. Se aplicó el Test de Bosco con los saltos Abalakov (ABK), Counter Mouvement Jump (CMJ) y Squat Jump (SJ) y el test Sprint Repeat Ability (RSA) (8x30), con pausas activas de $25^{\prime \prime}$, entre cada sprint. Son jugadores profesionales $(n=18)$, pertenecientes al equipo de Fútbol Sala profesional Sanpas Boyacá, con peso $67 \pm 6,996$, talla $172,89 \pm 6,859 \mathrm{~cm}$ y edad $23,89 \pm 3,248$. Los datos obtenidos, se analizaron con el coeficiente de correlación de Pearson ( $r$ ), para datos paramétricos y un Alpha mayor o igual a 0,05. Resultados: En el test de Bosco, en el salto Squat Jump, se presenta una media de 35,156 $\pm 4,5859 \mathrm{~cm}$; para el $A B K$, una media de 40,444 $\pm 5,0202$ y para CMJ, un valor de $36,050 \pm 4,2616$. Los resultados para el Test RSA, señala un índice de fatiga de 5,59 $\pm 2,811$. Conclusión: Se aceptó la hipótesis nula, considerando, de esta manera, que no hubo correlación entre la potencia y la resistencia a la velocidad.

Palabras claves: Fútbol Sala; Potencia; Resistencia a la velocidad; Test RSA; Test Bosco.

\section{ABSTRACT}

Introduction: Futsal is a collaborative-opposition sport, where members of the same team work to achieve an objective and at the same time interact with members of the opposing team. Their actions take place in a fast, changing and dynamic space and time. Objective: To investigate the correlation between power and speed endurance in professional players of futsal. Methodology: An analytical empirical approach and a descriptive correlational non-experimental design were used. The Bosco Test was applied with the Abalakov Jump (ABK), Counter Mouvement Jump (CMJ) and Squat Jump (SJ) and test Sprint Repeat Ability (RSA) (8×30) with active pauses 
of 25 " between each sprint. They are professional players $(n=18)$ belonging to the professional futsal team Sanpas Boyacá with weight $67 \pm 6.996$, height $172.89 \pm 6.859 \mathrm{~cm}$ and age $23.89 \pm 3.248$. The data obtained were analyzed with Pearson's correlation coefficient ( $r$ ) for parametric data and an Alpha greater than or equal to 0.05. Results: In the Bosco test in the Squat Jump a mean of $35.156 \pm 4.5859 \mathrm{~cm}$ is presented, for the ABK a mean of $40.444 \pm 5.0202$ and for CMJ a value of $36.050 \pm 4.2616$. The results for the RSA test showed a fatigue index of $5.59 \pm 2.811$.

Conclusion: The null hypothesis was accepted, thus considering that there was no correlation between power and speed endurance.

Keywords: Futsal; Power; Speed resistance; RSA Test; Test Bosco.

\section{INTRODUCCIÓN}

El Fútbol sala, se origina en Uruguay, en 1930. Su incorporación a la Federación Internacional de Fútbol Asociado (FIFA), se inició en el 2000 y es practicado por, alrededor, de 30 millones de personas, en el mundo. De las 209 federaciones afiliadas a la FIFA, 170 tienen liga profesional, entre ellas, la Federación Colombiana de Fútbol (FCF), donde se disputa la Liga Argos Futsal, en la cual, juega el equipo Sanpas Boyacá.

Se clasifica como un deporte de colaboración oposición (Álvarez Medina et al. 2002), donde los miembros de un mismo equipo trabajan para conseguir un objetivo (colaboración) y, al mismo tiempo, se relacionan con los miembros del equipo contrario (oposición); sus acciones, se desarrollan en un espacio y tiempo veloz, cambiante y dinámico (López Hierro, 2017), donde el jugador debe con o sin balón tomar decisiones para el desarrollo de las acciones técnicas y tácticas, en un corto lapso de tiempo. De ahí, que en la planificación se trabaje de forma integrada a la preparación física, técnica, táctica y psicológica (Agudelo, 2012).

Se caracteriza por la realización de movimientos veloces en línea recta o con cambio de dirección a máxima velocidad; los cambios ilimitados, el espacio reducido de juego y la detención del tiempo, cada vez que el balón abandona el juego o está en fuera de juego, hacen que el tiempo total de juego, reglamentariamente es de 40 minutos, se convierta en un tiempo total de 70 minutos 0 , incluso, de 75 a
80 minutos (Barbero, 2003), lo que genera, mantener una elevada intensidad de juego (Barbero, 2003). Ante estas circunstancias, los jugadores de Fútbol Sala, demandan el uso de vías energéticas aeróbicas y anaeróbicas (Álvarez Medina et al. 2002).

Es por ello, que se desarrolló una investigación descriptiva correlacional, teniendo como variables, la potencia de tren inferior y la resistencia a la velocidad en los jugadores del equipo de Fútbol Sala Sanpas Boyacá. Para medir la potencia, se realizó el test de Bosco, con los saltos de Abalakov (ABK), Squat Jump (SJ) y contamovement jump (CMJ), medidos en la plataforma de contacto Axón jump 2.0, la cual, permite obtener los datos a través del software v.4.04, conectado a un ordenador personal; de igual manera, la resistencia a la velocidad, se midió con el test de repeat sprint ability (RSA). Ambos test, se analizaron de forma individual y, luego, se estableció su correlación, a través de un paquete de datos estadístico SPSS v.22.0 (IBM Company Chicago). La presente investigación, se encuentra anexa al macroproyecto "Modelos y estrategias determinantes en el entrenamiento deportivo", de la maestría en Pedagogía de la cultura física de la Universidad Pedagógica y Tecnológica de Colombia-UPTC.

\section{MATERIALES Y MÉTODOS}

Participantes. En este estudio participaron 18 jugadores de futbol Sala, que pertenecen al equipo profesional de Sanpas Boyacá, con edades de 23,89 \pm 3,248 , peso $67 \pm 6,996 \mathrm{~kg}$, estatura $172,89 \pm 6,859 \mathrm{~cm}$ y un IMC de $22,39 \pm 1,62 \%$. Todos los participantes tenían una experiencia superior a los cinco años al entrenamiento de esta modalidad deportiva y entrenaban 4-5 sesiones por semana, durante el periodo de competición. Ninguno de los participantes en este estudio se encontraba lesionado en el momento de realizar las pruebas físicas. Todos los participantes firmaron el consentimiento informado antes de comenzar la investigación; además, se tuvo el consentimiento expreso por parte del club, en el cual, militan los deportistas.

Procedimientos. Esta investigación, se llevó a cabo en la finalización de la fase de grupos de la Liga de Futsal colombiana. Para este momento, el equipo estaba ubicado en la sexta casilla del grupo A; la fecha de realización de las pruebas fue consensuada con el cuerpo técnico del equipo, para interferir lo menos 
posible en el proceso de entrenamiento y competición. Los participantes fueron testeados en un solo día; en la primera parte de la sesión, se adelantó la charla informativa de los test que se realizarían (test de Bosco y test RSA); a continuación, el preparador físico del equipo efectuó un calentamiento general, con una carrera continua de 5 minutos y un calentamiento específico, enfocado en movimiento articulary Pliometría, con el fin de adaptar al deportista en los dos test: uno de potencia en tren inferior y el segundo de resistencia a la velocidad.

Se utilizaron los parámetros determinantes del test de Bosco, para la variable potencia. De éste, se tomaron las variables dependientes de los saltos Squat Jump (SJ), Counter mouvement Jump (CMJ) y Abalakov (ABK). Las variables analizadas dentro de estos saltos fueron: altura del salto, despegue y la potencia medida en watts. Para llevar a cabo el análisis se utilizó una plataforma de contacto (Axón
Jump). Para el desarrollo de cada salto, se tomaron 3 repeticiones de cada tipo de salto, con un descanso de 40 segundos entre ellos; primero se realizó el SJ, luego, el CMJ y, por último, el ABK.

Respecto a la variable de resistencia a la velocidad, se utilizó el test Repeat Sprint Ability (RSA) (Figura 1); para analizar esta capacidad de repetir sprint, se utilizó el protocolo establecido por Spencer et al. (2011). El test consistía en realizar 8 sprint, con una distancia de 30 metros a máxima intensidad, con una recuperación de 25 segundos entre cada sprint, tiempo, en el cual, los jugadores invertían en volver al punto de salida y prepararse para el siguiente sprint. Se tomaron las siguientes variables del test para realizar el análisis estadístico: tiempo de cada sprint, promedio de tiempo de los 8 sprint, mejor tiempo y el índice de fatiga, que se calculó, atendiendo a la fórmula propuesta por Barbero-Álvarez et al. (2010).

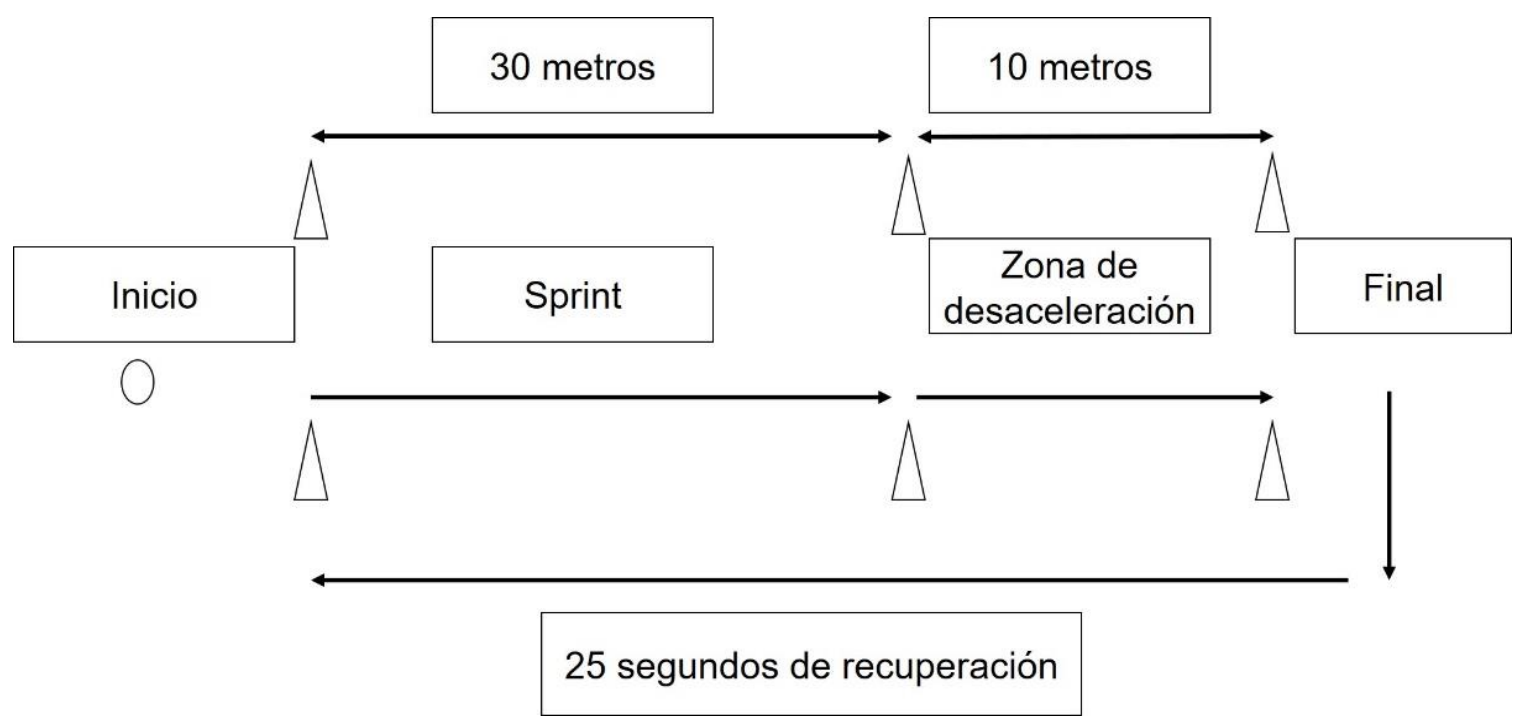

Figura 1. Test Sprint Repeat Ability-RSA

Análisis estadístico. En palabras de Hernández et al. (2014), el objetivo de los estudios de tipo correlacional es establecer el grado de relación que existe entre dos o más variables de estudio. En primer lugar, se realizará un análisis de la caracterización de los deportistas estudiados; después, se adelantará un análisis descriptivo de cada una de las variables de estudio que, para la presente investigación, son la Resistencia a la velocidad y la Fuerza Explosiva de tren inferior, evaluada desde el Squat Jump, el Counter Movement Jump y el Abalakov. Luego de establecer las características principales de las variables, se procede a establecer el grado de relación de las mismas en los jugadores de categoría sub- 20, de Patriotas Boyacá.
Dentro del proceso llevado a cabo, se analiza la estadística descriptiva de la caracterización del grupo de estudio, en donde se estudiarán aspectos, como talla, peso, edad y el índice de masa corporal, con su respectiva clasificación; además, se establece, en segundo lugar, la evaluación y el análisis de los test de fuerza explosiva que, para el caso, corresponden al Squat Jump (SJ), Counter Movement Jump (CMJ) y Abalakov (ABK); estos test, se realizan en la plataforma Axón Jump, que permite obtener los datos, gracias a su software (v.4.04), el cual, se instaló en un computador personal. Luego de obtener los datos de la plataforma de contacto, los datos fueron exportados a Microsoft Excel, para su tratamiento y 
posterior egreso al paquete estadístico SPSS v.22.0 (IBM Company Chicago), para su interpretación y respectivo análisis.

También, se llevó a cabo el Test de Repeat Sprint Ability, donde se busca índice de fatiga, un indicador directo de la resistencia a la velocidad de los deportistas evaluados; los datos obtenidos, se sistematizaron en Microsoft Excel, en una hoja de cálculo, diseñada para el test en mención. De allí, se exportaron los resultados del índice de fatiga al paquete estadístico SPSS v.22.0 (IBM Company Chicago), para su análisis.

\section{RESULTADOS Y DISCUSIÓN}

En la tabla 1, se encuentra que, para el mejor tiempo, se registran valores de CV de 0,189\%, lo que indica que los resultados, para el grupo de estudio, son homogéneos. En cuanto al peor tiempo, el CV es de $0,246 \%$, lo que demuestre que, peor tiempo, los datos son casi parecidos. Por otro lado, al analizar el índice de fatiga, el CV es de 0,5028 \%, lo que demuestra que los datos son heterogéneos, debido a que no todos los deportistas están en un estado excelente, de acuerdo con la clasificación general del test.

Tabla 1. Resultados Test Sprint Repeat Ability-RSA

\begin{tabular}{|c|c|c|c|}
\hline $\begin{array}{c}\text { Estadísticos } \\
\text { descriptivos }\end{array}$ & Mejor tiempo & Peor tiempo & Índice de fatiga \\
\hline $\mathrm{N}$ & 18 & 18 & 18 \\
\hline Promedio & 4,45 & 4,93 & 5,59 \\
\hline Mediana & 4,39 & 4,86 & 5,30 \\
\hline $\begin{array}{c}\text { Desviación } \\
\text { estándar }\end{array}$ & 0,189 & 0,246 & 2,811 \\
\hline Mínimo & 4,17 & 4,63 & 2,26 \\
\hline Máximo & 4,89 & 5,69 & 12,30 \\
\hline
\end{tabular}

En la tabla 2, en cuanto a potencia se refiere, se encuentran los resultados obtenidos en el test de Bosco; en primer lugar, el Squat Jump, los valores de potencia, se obtuvieron con una media de 2752,29 $\pm 342,69$ watts, con un CV de 12,45 \%, lo cual, demuestra un grupo casi homogéneo, con valores leves de dispersión y con un rango de 1303,70 watts.

Tabla 2. Resultados Test de Bosco.

\begin{tabular}{|c|c|c|c|c|c|}
\hline $\begin{array}{c}\text { Estadísticos } \\
\text { descriptivos }\end{array}$ & $\mathbf{N}$ & Mínimo & Máximo & Media & $\begin{array}{c}\text { Desviación } \\
\text { estándar }\end{array}$ \\
\hline $\begin{array}{c}\text { Test de } \\
\text { Potencia SJ } \\
\text { (watts) }\end{array}$ & 18 & 2029,90 & 3333,60 & 2752,03 & 342,69 \\
\hline $\begin{array}{c}\text { Test de } \\
\text { potencia } \\
\text { CMJ (watts) }\end{array}$ & 18 & 2223,90 & 3401,70 & 2821,29 & 334,18 \\
\hline $\begin{array}{c}\text { Test de } \\
\text { potencia } \\
\text { ABK (watts) }\end{array}$ & 18 & 2347,70 & 3651,40 & 3093,75 & 376,90 \\
\hline $\begin{array}{c}\text { N válido (por } \\
\text { lista) }\end{array}$ & 18 & & & & \\
\hline
\end{tabular}


En cuanto al CMJ,se puede evidenciar que el grupo evaluado presenta una media de 2821,29 $\pm 334,18$ watts, lo que indica un CV de $11,84 \%$, mostrando una leve dispersión en el grupo de estudio, con un rango de 1177,80 watts, lo cual, indica que el grupo es casi homogéneo; de igual forma, ocurre con el ABK, donde se halló una media de 3093,75 \pm 376,90 watts, lo que expresa un CV de $12,18 \%$ y un rango de 1303,7 watts, lo cual, indica que el grupo, en esta prueba, es casi homogéneo.

Esto refleja que los datos obtenidos en los tres diferentes saltos, para este grupo de estudio, en general, son casi homogéneos, es decir, casi parecidos, evidenciando, de esta manera, que el entrenamiento, llevado a cabo por el cuerpo técnico del equipo Sanpas Boyacá, se ha desarrollado de manera igualitaria en todos los jugadores. Con relación a lo anterior, es necesario valorar los resultados obtenidos por los deportistas, en cuanto a la potencia; se determina, si existe prevalencia en las manifestaciones activas o reactivas de la fuerza. Se observa que en los deportistas de este estudio predomina la expresión reflejo elástica explosiva, porque es la capacidad contráctil y el reclutamiento de unidades motoras, se reflejan en cada salto, mientras que la reutilización de la energía elástica, no es contundente en los resultados de los saltos.
Finalmente, se puede decir que prevalece el componente reactivo, dada la combinación del componente contráctil, las capacidades de reclutamiento y sincronización de fibras, el componente elástico y el reflejo, aunados al movimiento oscilante, generado por los brazos, lo cual, aporta en alto grado al desarrollo de la potencia de tren inferior.

\section{Correlación entre el índice de fatiga que representa} la resistencia a la velocidad y los test utilizados para hallar la potencia de tren inferior de los deportistas de la selección masculina de SANPAS Boyacá. En cuanto a la relación con el $\mathrm{SJ}$, se halló una $r=-0,18$ y un $p$ valor de 0,47 (para todos el $p \leq 0,05$ ), lo cual, indica que existe una correlación débil e inversa; respecto al CMJ, se halló una $r=-0,38$ y un $p$ valor de 0,12 , que sugiere que existe una relación débil e inversa con la resistencia a la velocidad y, por el último, el $A B K$, se evidencia que existe una relación débil e inversa de las variables, debido a que se obtuvo una $r$ de-0,17, con un $p$ valor de 0,49 .

En la figura 2, se obtiene un coeficiente de determinación de un $3 \%$, lo cual, indica que, tan solo ese porcentaje de cambios en resistencia a la velocidad, se deben a la fuerza explosiva de tren inferior de los deportistas.
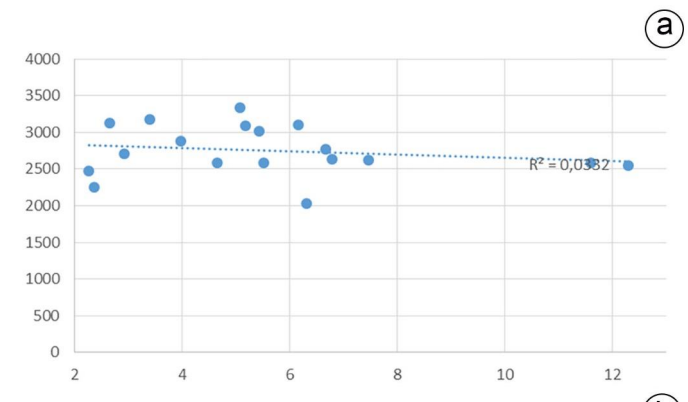

(b)
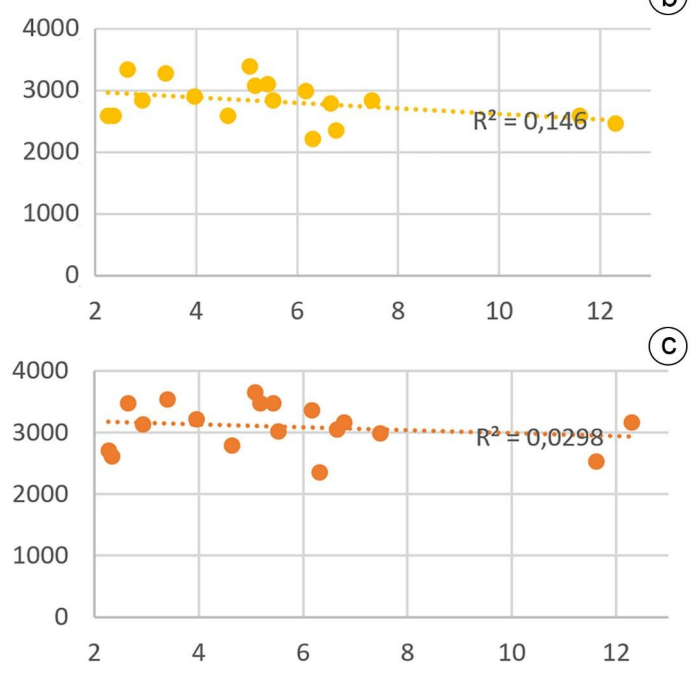

Figura 2. Coeficientes de determinación. a. Squat Jump-SJ; b. Counter Mouvement Jump-CMJ; c. Abalakov-ABK. 
Se evidencia un coeficiente de relación de un $15 \%$, que señala que solo ese porcentaje de los cambios de la resistencia a la velocidad, se deben a la capacidad elástico explosivo de tren inferior de los deportistas.

Al observar el caso del salto Abalakov, se evidencia que tan solo el coeficiente de correlación fue de un $3 \%$.

Se acepta la hipótesis nula, que indica que no existe relación entre resistencia a la velocidad y la manifestación elástico explosiva de la fuerza. Los resultados demuestran que, en el caso de los deportistas de Fútbol Sala, de género masculino, del equipo SANPAS Boyacá, residentes en la ciudad de Tunja, no existe relación entre la resistencia a la velocidad y las manifestaciones explosiva, elástica explosiva y reflejo elástico explosiva de tren inferior; de allí, se establece la necesidad de evaluar otras variables que puedan incidir directamente sobre la resistencia a la velocidad de los deportistas.

Al indagar en diferentes fuentes de información, como bases de datos, referente a investigaciones sobre Futsal, en cuanto a correlación entre resistencia a la velocidad (RSA) y potencia en miembros inferiores con los saltos de SJ, CMJ y ABK, en el género masculino, solo se encontró un artículo, que se acercó al interés de esta investigación, aunque carece de datos en cuanto al salto $A B K$ y resultados más específicos del RSA.
Dentro de las características del presente estudio, se establece la prueba RSA, con 30 metros de distancia, en 8 sprint y un descanso activo de 25 segundos y su correlación con SJ, CMJ y ABK, para masculinos; una de las investigaciones que está en la misma línea es la de Sánchez et al. (2014), quienes correlacionaron el RSA con SJ y CMJ, en hombres que, por un lado, son deportistas que participan en la Liga Argos Futsal de Colombia y, por el otro, son atletas que participan en la 2B de futsal de España.

Es de resaltar los resultados homogéneos que se encuentran en ambos estudios, en cuanto a la edad, el peso y la talla; específicamente, los participantes boyacenses, en edad, reflejan $23,89+3,248$ vs mientras que sus pares españoles están en 23,73 \pm 5,55 años. Asimismo, en cuanto a peso, se observa $67 \pm 6,99$ vs $69,91 \pm 8,47 \mathrm{~kg}$ y, por último, la talla, $172,89 \pm 6,859$ vs $172,27 \pm 6,62$, lo que indica el gran parecido en ambos grupos.

Al abordar el aspecto correlacional (Tabla 3), Sánchez et al. (2014) manifiestan que en cuanto al CMJ no se encontraron correlaciones significativas con el RSA, suceso que se evidencia en este estudio, donde el resultado del $\mathrm{CMJ}$ arrojó una $\mathrm{r}=-0,38$ y un $\mathrm{p}=0,12$, lo que indica que existe una relación débil e inversa con la resistencia a la velocidad.

Tabla 3. Correlaciones.

\begin{tabular}{|c|c|c|c|c|c|}
\hline \multicolumn{2}{|c|}{ Correlaciones } & $\begin{array}{c}\text { Índice de } \\
\text { fatiga } \\
\text { (\%) }\end{array}$ & $\begin{array}{c}\text { Test de } \\
\text { potencia } \\
\text { SJ } \\
\text { (watts) }\end{array}$ & $\begin{array}{c}\text { Test de } \\
\text { potencia } \\
\text { CMJ } \\
\text { (watts) }\end{array}$ & $\begin{array}{c}\text { Test de } \\
\text { potencia } \\
\text { ABK } \\
\text { (watts) }\end{array}$ \\
\hline \multirow{2}{*}{$\begin{array}{c}\text { Índice de } \\
\text { fatiga (\%) }\end{array}$} & $\begin{array}{c}\text { Correlación } \\
\text { de Pearson }\end{array}$ & 1,00 & $-0,18$ & $-0,38$ & $-0,17$ \\
\cline { 2 - 6 } & $\begin{array}{c}\text { Sig. } \\
\text { (bilateral) }\end{array}$ & & 0,47 & 0,12 & 0,49 \\
\cline { 2 - 6 } & $\mathrm{N}$ & 18,00 & 18,00 & 18,00 & 18,00 \\
\hline
\end{tabular}


Por otra parte, los resultados del equipo español, en lo que se refiere a SJ, sí arroja valores significativos en cuanto al RSA, al correlacionarlo con el mejor Sprint, donde se encontró una $r=0.676 ; p=0.022$, indicando una relación moderada fuerte; caso contrario al de los deportistas boyacenses, en quienes se obtuvieron resultados de $r=-0,38 ; p=0,12$, indicando una relación débil e inversa.

Desde otra perspectiva, Dal Pupo et al. (2017) modificaron el test, dejando 6 sprint de 25 metros por 25" de descanso activo, afirmando que, en este estudio, también se encuentra una relación significativa entre el SJ, el CMJ, el tiempo medio y mejor sprint de la prueba RSA; sin embargo, los datos de Sánchez et al. (2014) y Dal Pupo et al. (2013), no aportan mucho a la discusión, entendiéndose que, al hablar del mejor sprint, se traduce en un esfuerzo de alta intensidad, en un espacio y tiempo corto, diferente a las exigencias del test RSA.

Aun así, llama particularmente la atención los resultados obtenidos en el SJ, donde expone valores de 40,51 $\pm 5,38$, en sub 17 y, 37,75 $\pm 2,40$, en sub 15 e, igualmente, el estudio de Massuça et al. (2015), muestra valores de 36,41 $\pm 8,06 \mathrm{~cm}$, mientras que en la presente investigación, se hallaron resultados de 35,15 $\pm 4,58$, evidenciándose mejores resultados en el anterior estudio, teniendo en cuenta, que se tratan de categorías inferiores. Esto se podría presentar, aparentemente, porque las cargas de fuerza dosificadas a los jugadores de Sanpas Boyacá no están surtiendo el efecto esperado.

En esta investigación, se puede apreciar que el resultado para el CMJ, en los atletas del equipo de Fútbol Sala FIFA Sanpas Boyacá, es de 36,05 \pm 4,26, al relacionar este resultado con otros estudios; por ejemplo, Dal Pupo et al. (2017) describen saltos de 45,71 $\pm 5,05$, para Sub 17 y, 41,50 $\pm 5,56$, para sub 15 , por su parte, Silva et al. (2012) exponen $43,8 \pm 6,8$.

Del mismo modo, Mendonça et al. (2017) evalúan el $\mathrm{CMJ}$ en tres momentos diferentes, de un encuentro en deportistas de Fútbol Sala sub-17, del campeonato Romairense, con resultados de: antes del encuentro, $39,1 \pm 5,28$; en el medio tiempo, 36,7 $\pm 4,52$ y, luego del encuentro, un valor de $35,9 \pm 5,51$, encontrándose que son valores mucho más altos que los de los Boyacenses.
Y, por último, Vasconcelos et al. (2020) realiza una prueba de salto de SJ y CMJ sin estímulos motivacionales (SM) y con estímulos motivacionales (CM); los resultados arrojan que, al incluir estímulos motivacionales, los resultados de los saltos mejoraron SJ (SM 32,11 $\pm 4,95 ; C M ~ 35,35 \pm 4,04 ; P<0,01)$ y en el CMJ (SM 34,76 $\pm 5,25 ; C M ~ 36,51 \pm 4,48 ; P=0,04)$.

Finalmente, al hablar de salto Abalakov, en el estudio de Centeno (2013), se analizó el salto en jugadores de Fútbol Sala, dando como resultado 33,62 \pm 7,02, mientras que, en el presente estudio, el resultado fue de $40,44 \pm 5,02$.

Aunque los test cuentan con unos protocolos y condiciones establecidas para garantizar la fiabilidad, Vasconcelos et al. (2020) describen que un estímulo motivacional puede influir en obtener mejores resultados en los saltos del test de Bosco y Mendonça et al. (2017) muestran que no necesariamente el deportista debe tener un tiempo de descanso para lograr buenos resultados, toda vez que, en su estudio, se evidenció que, al hacer las comparaciones antes-durante, antes-después y durante-después del partido, los deportistas no tuvieron caída en el rendimiento de las evaluaciones.

Al no existir una correlación entre la fuerza explosiva, medida a través de los saltos de SJ, CMJ y Abalakov, con la resistencia a la velocidad, es oportuno considerar otras variables, que influyan en el rendimiento en la RSA, así como la contribución de la fuerza explosiva en el rendimiento en RSA. Barbero \& Castagna (2005) sustentan que algunas de las variables que pueden influir en el rendimiento de la RSA, se relacionan con la condición física aeróbica, en términos fisiológicos, en cuanto a la capacidad del atleta, en el proceso de resíntesis de $\mathrm{PCr}$ y el "aclarado" de lactato, debido a que, el proceso de resíntesis de $\mathrm{PCr}$, se hace en presencia de oxígeno, por lo cual, sería importante que futuros estudios se encarguen de estudiar la relación de la capacidad aeróbica con la capacidad para repetir sprint.

Asimismo, se debe considerar, desde el punto de vista fisiológico, si el tipo de fibra muscular (lentas o rápidas) influye en rendimiento en el RSA, como también los valores obtenidos en las pruebas de salto; en este caso, los realizados por medio del test de Bosco (SJ, CMJ, ABK), como también el tipo de suministro energético. 
A la luz de las anteriores investigaciones es oportuno considerar que no hay correlación entre las variables SJ, CMJ, ABK y el índice de fatiga o RSA. Siendo así, es pertinente estimar que la potencia y la resistencia a la velocidad, se deben entrenar de manera independiente dentro de la planificación, que puede brindar mejores resultados en el rendimiento; desde los diferentes componentes del entrenamiento deportivo (Agudelo, 2012), entrenar la resistencia a la velocidad y la fuerza, se puede realizar de forma simultánea y ambas mejoran (Torres-Torrelo et al. 2018). Es importante tener en cuenta todos los aspectos desde lo físico hasta lo motivacional.

\section{CONCLUSIONES}

1. En primer lugar, la hipótesis muestra que no existe co-relación entre la potencia de tren inferior y la resistencia a la velocidad de los jugadores de Fútbol Sala FIFA masculino, del equipo SANPAS Boyacá, de Tunja, por lo que se acepta la hipótesis nula.

Este resultado podría estar condicionado por varios aspectos. Por una parte, por el tipo de proceso metabólico implícito en cada uno de los atletas al momento de afrontar una serie de sprint repetidos, usando, comúnmente, la vía anaeróbica, con baja presencia de lactato en esfuerzos cortos y rápidos y la vía aeróbica, con presencia moderada de lactato en esfuerzos más prolongados (Sánchez et al. 2005). Por otro lado, aparecen las características físicas, donde se involucran el tipo de fibra muscular, que actúa en el gesto (fibras rojas o lentas, fibras blancas y rápidas) (Verjorhanski, 1990) y la capacidad que tiene ese músculo o grupo de músculos para realizar una acción en el menor tiempo (Mazón et al. 2017). Y, por último, las características fisiológicas que se relaciona con la capacidad neuromuscular que posee el atleta, para generar una alta velocidad de contracción, ante una resistencia determinada (Rodríguez, 2007).

2. Con relación al perfil de fuerza explosiva de tren inferior de los deportistas, evidencia prevalencia de la capacidad reflejo elástico explosivo sobre la capacidad elástico explosivo y la capacidad contráctil de los deportistas. Para el caso de la capacidad contráctil, se evidencia que, en los deportistas del equipo, prevalece sobre la capacidad elástica, esto se debe a la posición de juego y el papel de cada uno de los deportistas en competencia; sin embargo, es necesario llevar a cabo una investigación que permita establecer si dicha asociación, entre el perfil de potencia y la posición de juego en el fútbol sala, es verídica.
3. Al analizar la evaluación de la resistencia a la velocidad, se encuentra que el test se ajusta a las características de deportes intermitentes, como el caso del Fútbol Sala, donde la secuencia de movimientos en el RSA, se asemeja a las carreras que el deportista realiza en el juego, representados en esfuerzos máximos intermitentes, con espacios cortos de recuperación, generalmente, activa. En cuanto a la planificación y la dosificación del entrenamiento en resistencia a la velocidad, el cuerpo técnico del equipo Sanpas Boyacá, debe tener en cuenta los resultados obtenidos por sus atletas, debido a que presentan valores excelentes, en un $89 \%$ de la muestra y valores buenos, en un $11 \%$, del grupo de estudio.

Conflicto de intereses: El manuscrito fue elaborado y revisado por los dos autores, quienes declaran la ausencia de cualquier conflicto, que pueda poner en riesgo la validez de los resultados.

\section{REFERENCIAS}

1. AGUDELO, C. 2012. Planificación del entrenamiento deportivo por modelamiento. Principios, estructuras y metodología general. Kinesis (Armenia, Colombia). 156p.

\section{2.ÁLVAREZMEDINA,J.; GIMÉNEZ, SALILLASL.;CORONA} VIRÓN, P.; MANONELLES MARQUETA, P. 2002. Cardiovascular and metabolic necessities of indoor football: analysis of the competition. Apunts educación física y deportes. 67:45-51.

3. BARBERO, C. 2003. Quantitative analysis of temporal dimension for competition in football. Europan Journal of Human Movement. 10(1):143-163.

4. BARBERO, J.C.; CASTAGNA, C. 2005. El test Yo-Yo de recuperación intermitente nivel 1 . Revista de entrenamiento Deportivo. 2:21-27.

5. BARBERO-ÁlVAREZ, J.; COUNTTS, A.; GRANDA, J.; BARBERO-ÁlVAREZ, V.; CASTAGNA, C. 2010. The validity and reliability of a global positioning satellite system device to assess speed and repeated sprint ability (RSA) in athletes. J Sci Med Sport. 13(2):232-235. https://doi.org/10.1016/j.jsams.2009.02.005

6. CENTENO, R. 2013. Valores de referencia para saltos en plataforma dinamométrica en una población de deportistas andaluces. Documento presentado 
para obtener el título de Doctorado. Universidad de Olavide, Sevilla, España.

7. DAL PUPO, J.; DETANICO, D.; BUDAL, F.; NASCIMENTO, P.; GUGLIELMO, L.;DOSSANTOS, S. 2017. Capacidade de sprints repetidos e níveis de potencia muscular em jogadores de futsal das categorías sub-15 e sub-17. Revista brasileira de ciencias do esporte. 39:73-78. https://doi.org/10.1016/j.rbce.2016.01.010

8. DAL PUPO, J.; DETANICO, D.; CARMINATTI, L.; SANTOS, S. 2013. Respuestas fisiológicas y neuromusculares en sprints repetidos con cambios de dirección y en línea recta. Apunts sports medicine. 48(178):43-48. https:// doi.org/10.1016/j.apunts.2012.11.001

9. HERNÁNDEZ SAMPIERI, R.; FERNÁNDEZ COLLADO, C.; BAPTISTA LUCIO, P. 2014. Metodología de la investigación. 6ta Edición. McGraw Hill (México D.F. México). 634p.

10. LÓPEZ HIERRO, J.V. 2017. Manual de la UEFA para entrenadores de fútsal. Union des Associations Européennes de Football (UEFA). 202p.

11. MASSUÇA, L.M.; HONÓRIO, S.A.A.; MONTEIRO, L.F.; BATISTA, M.A.S.; SOUSA, P.M.D. 2015. Impacto da morfologia, força e potência dos membros inferiores na agilidade de atletas profissionais de futsal. RBFF-Revista Brasileira de Fútsal e Futebol. 7(23):72-79.

12. MAZÓN, O.D.; OBREGÓN, G.A.; CAIZA, M.V.; BAYAS, J.C.; BAYAS, R.F. 2017. Análisis de la fuerza explosiva en el golpe de balón con el pie en los futbolistas categoría senior de la universidad nacional de Chimborazo - ecuador. Estudio de caso: test de salto horizontal. European Scientific Journal. 13(18):115-139. https:// doi.org/10.19044/esj.2017.v13n18p115

13. MENDONÇA, W.; SOUZA, S.; SILVA, F.; SANTOS, R.; SANTA CRUZ, R. 2017. Potência muscular de membros inferiores em diferentes momentos de umjogo oficialde futsal. Revista De Educação Física / Journal of Physical Education. 86(2). https://doi.org/10.37310/ref.v86i2.216
14. RODRÍGUEZ, P. 2007. Fuerza, su clasificación y pruebas de valoración. Revista de la Facultad de Educación, Universidad de Murcia. 2-10.

15. SÁNCHEZ, J.; BORES, A.; RODRÍGUEZ, A.; GARCÍA, D.; GUILLÉN, J.; ROMO, D.; PAZ, A. 2014. Evaluación de la relación entre la habilidad de repetir sprint (RSA) tanto en línea recta como con cambios de dirección, y la fuerza explosiva del tren inferior en jugadores de élite de fútbol sala. VIII Congreso Internacional de la Asociación Española de Ciencias del Deporte.

16. SÁNCHEZ-SÁNCHEZ, J.; BLÁZQUEZHERNÁNDEZ, F.; GONZALO MARTÍN, A.; YAGÜE CABEZÓN, J.M. 2005. La resistencia a la velocidad como factor condicionante del rendimiento del futbolista. Apunts Educación Física y Deportes. 81:47-60.

17. SILVA, J.; DETANICO, D.; FLORIANO, L.; DITTRICH, N.; NASCIMENTO, P.; SANTOS, S.; GUGLIELCO, L.; 2012. Níveis de potência muscular em atletas de futebol e futsal em diferentes categorías e posições. Motricidade. 8(1):14-22. https:// doi.org/10.6063/motricidade.8(1).233

18. SPENCER, M.; PYNE, D.; SANTISTEBAN, J. 2011. Fitness determinants of Repeat Sprint Ability in highly trained youth football players, international journal of sports physiology of performance. 6:497-508.

19. TORRES-TORRELO, J.; RODRÍGUEZ-ROSELL, D.; MORA-CUSTODIO, R.; PAREJA-BLANCO, F.; YÁÑEZ-GARCÍA, J.; GONZÁLEZ-BADILLO,J. 2018. Effects of Resistance Training and Combined Training Program on Repeated Sprint Ability in Futsal Players. Int J Sports Med. 39(07):517526. https://doi.org/10.1055/a-0596-7497

20. VASCONCELOS, A.B.S.; FARINON, R.; PERASOL, D.; SANTOS, J.M.M. DOS.; FREITAS, V.H. DE. 2020. Influência de estímulos motivacionais no desempenho em testes de salto vertical de atletas de futsal sub17. Revista Brasileira De Educação Física E Esporte. 34(4):727-733. https://doi. org/10.11606/1807-5509202000040727

21. VERJORHANSKI, I. 1990. Entrenamiento deportivo: planificación y programación. Martínez Roca (Barcelona, España). 166p. 